\title{
Management of Patients With Hypertensive Urgencies and Emergencies
}

\author{
A Systematic Review of the Literature \\ David Cherney, MD, Sharon Straus, MD, FRCPC
}

\begin{abstract}
BACKGROUND: Hypertensive urgencies and emergencies are common clinical occurrences in hypertensive patients. Treatment practices vary considerably to because of the lack of evidence supporting the use of one therapeutic agent over another. This paper was designed to review the evidence for various pharmacotherapeutic regimens in the management of hypertensive urgencies and emergencies, in terms of the agents' abilities to reach predetermined "safe" goal blood pressures (BPs), and to prevent adverse events.
\end{abstract}

METHODS: MEDLINE was searched from 1966 to 2001, and the reference lists of all the articles were retrieved and searched for relevant references, and experts in the field were contacted to identify other relevant studies. The Cochrane Library was also searched. Studies that were eligible for inclusion in this review were systematic reviews of randomized control trials (RCTs) and individual RCTs, all-ornone studies, systematic reviews of cohort studies and individual cohort studies, and outcomes research. No language restrictions were used.

RESULTS: None of the trials included in this review identified an optimal rate of BP lowering in hypertensive emergencies and urgencies. The definitions of hypertensive emergencies and urgencies were not consistent, but emergencies always involved target end-organ damage, and urgencies were without such damage. Measures of outcome were not uniform between studies. The 4 hypertensive emergency and 15 hypertensive urgency studies represented 236 and 1,074 patients, respectively. The evidence indicated a nonsignificant trend toward increased efficacy with urapidil compared to nitroprusside for hypertensive emergencies (number needed to treat [NNT] for urapidil to achieve target BP, 12; $95 \%$ confidence interval $[95 \% \mathrm{CI}]$, number of patients needed to harm [NNH], 5 to NNT, 40 compared to nitroprusside). Several medications were efficacious in treating hypertensive urgencies, including: nicardipine (NNT for nicardipine compared to plabebo, 2 in one study [95\% CI, 1 to 5] and 1 in another [95\% CI, 1 to 1]); lacidipine (NNT, 2; 95\% CI, 1 to 8 for lacidipine vs nifedipine) or urapidil (NNT for urapidil compared to enalaprilat and nifedipine, $4 ; 95 \% \mathrm{CI}, 3$ to 6 ); and nitroprusside and

Received from the Department of Medicine, Toronto General Hospital, University of Toronto, Toronto, Ontario, Canada.

Address correspondence and requests for reprints to Dr. Cherney: 50 Walmer Rd., Apt. 107, Toronto, Ontario, M5R 2X4, Canada (e-mail: dchern@hotmail.com). fenoldopam (all patients reached target BP in 2 studies). The studies reported 2 cases of cerebral ischemia secondary to nifedipine.

CONCLUSIONS: Many effective agents exist for the treatment of hypertensive crises. Because of the lack of large randomized controlled trials, many questions remain unanswered, such as follow-up times and whether any of the studied agents have mortality benefit.

KEY WORDS: hypertensive urgency; hypertensive emergency; hypertensive crisis.

J GEN INTERN MED 2002;17:937-945.

$\mathbf{H}$ ypertensive urgencies and emergencies are common clinical occurrences that may account for as many as $27.5 \%$ of all medical emergencies presenting to the emergency department ${ }^{1}$ and $3 \%$ of all emergency room visits, ${ }^{2}$ and that may affect as many as $1 \%$ of hypertensive patients. ${ }^{3,4}$ However, clinical treatment practices for the management of hypertensive urgencies and emergencies vary considerably. ${ }^{1}$ This practice variability is in part because of the lack of evidence supporting the use of one therapeutic agent over another. This paper was designed to review the evidence for various pharmacotherapeutic regimens in the management of hypertensive urgencies and emergencies in terms of the agents' ability to reach a predetermined "safe" target blood pressure (BP) and to prevent adverse events.

For this paper, we used the following definitions for hypertensive urgencies and emergencies, which were taken from the literature: in a hypertensive emergency, a patient has evidence of target end-organ damage, such as encephalopathy, unstable angina, stroke, or a dissecting aortic aneurysm. The absolute level of BP in this situation is not as important as the evidence of end-organ damage. ${ }^{1}$ In hypertensive urgencies, the patient has elevated BP but has no evidence of end-organ damage.

\section{METHODS}

\section{Search Strategy}

We searched MEDLINE from 1966 to 2001 using the terms hypertensive urgency, hypertensive emergency, hypertensive crisis, uncontrolled hypertension, refractory 
hypertension, poorly responsive hypertension, poorly responsive blood pressure, and malignant hypertension. We also used search terms for finding systematic reviews. ${ }^{5} \mathrm{We}$ then retrieved the references of all the articles and searched the bibliographies for additional relevant references. Experts in the field were contacted to identify any relevant studies. We also searched the Cochrane Library using the terms hypertension and malignant hypertension. Studies that were eligible for inclusion in this review were systematic reviews of randomized control trials (RCTs) and individual RCTs, all-or-none studies, systematic reviews of cohort studies and individual cohort studies, and outcomes research, i.e., Level 1 or 2 evidence. We did not include any language restrictions in the literature search. Our study included all classes of antihypertensive agents. The agents could have been given via sublingual (SL), oral (PO), or parenteral (IV) routes, depending on the agent and the setting.

The articles were appraised by 2 independent reviewers who assessed their level of evidence on the basis of the definitions that can be found in Table 1. Levels of evidence are useful in assessing the validity of evidence and in interpreting evidence. They have been designed to identify the specific methods that maximize the validity of a study's conclusions and structure them into a hierarchy of study types with the most valid at the forefront. ${ }^{6}$ Only those articles with Level 1 or 2 evidence were included in this review. Number needed to treat (NNT) and relative risk (RR) calculations were performed using the Mount Sinai Hospital Center for Evidence Based Medicine statistics calculator $^{7}$ and were included for comparative purposes. The NNT calculations were given, when possible, for the most effective agent in trials comparing more than 1 antihypertensive. The RR calculations were also performed, when possible, to give an estimate of the likelihood of the less-effective agent reaching the target BP.

Table 1. Levels of Evidence/Therapy Studies ${ }^{6}$

\begin{tabular}{ll}
\hline \hline Level of Evidence & \multicolumn{1}{c}{ Study Design } \\
\hline $1 \mathrm{a}$ & Systematic review of RCTs \\
$\mathrm{lb}$ & Individual RCT \\
$\mathrm{lc}$ & All or none \\
$\mathrm{c} \mathrm{a}$ & Systematic review of cohort studies \\
$\mathrm{2b}$ & Individual cohort study \\
$\mathrm{2c}$ & Outcomes research ${ }^{6, \dagger}$ \\
$\mathrm{3a}$ & Systematic review of case control study \\
$3 \mathrm{~b}$ & Individual case control study \\
4 & Case series \\
5 & Expert opinion, consensus meeting \\
\hline
\end{tabular}

* Met when all patients died before the treatment became available, but some now survive on it; or when some patients died before the treatment became available, but none now die on it.

† Outcomes research uses data to understand how well treatments work in the real world, in specific patient populations and under specific conditions (i.e., the end result of healthcare practices and interventions).

$R C T$, randomized control trial.

\section{Study Participants}

Study participants were over the age of 18 years and had had a hypertensive emergency or urgency at the time of their enrollment in the study. Exclusion criteria were varied and included the very elderly ( $>80$ years old), ${ }^{8,9}$ pregnancy and lactation, ${ }^{8-19}$ history of organ transplantation, ${ }^{8,17}$ immunosuppression, ${ }^{17-19}$ acute ${ }^{8,9,17,18}$ or chronic renal failure, ${ }^{8,11,12,17,18,20}$ dialysis, ${ }^{17-19}$ valvular heart disease, ${ }^{11,12,14,21}$ recent stroke, ${ }^{11,12,14,21,22}$ acute myocardial infarction, ${ }^{9,11,12,14-16,21}$ coronary bypass surgery or congestive heart failure, ${ }^{11,12}$ "bilateral stenosis"9 or arrhythmia, ${ }^{15-20}$ or a known secondary cause of hypertension such as pheochromocytoma. ${ }^{8,17-19}$ Other exclusion criteria included hypothyroidism, ${ }^{19}$ hepatic $^{14,15,17-19}$ or hematological disease, ${ }^{14,17,18}$ asthma or chronic obstructive pulmonary disease, ${ }^{16}$ alcohol intoxication, ${ }^{12}$ parenteral analgesia, ${ }^{17,18}$ dopamine antagonists ${ }^{17-19}$ or "considerable pain." 13 Patients with signs of end-organ involvement were excluded in the hypertensive urgency studies. Signs of end-organ involvement (acute myocardial infarction, aortic dissection, and focal neurological deficits) aside from hypertensive changes in the retina were exclusion criteria in 1 study of hypertensive emergencies. ${ }^{10}$

\section{RESULTS}

Six hundred hypertensive urgency or hypertensive emergency abstracts were identified in the literature. Most of these studies were excluded because they were nonhuman studies, did not involve patients with high enough BP to qualify as an urgency or an emergency, were safety/ tolerability studies, or were case-series or case reports. We were left with 39 studies after excluding all of the above. Ten studies were then excluded because they did not include a target BP and were therefore of limited usefulness to clinicians and could not be compared to other agents in terms of NNT or RR. Other studies were excluded because they were methodologically flawed in their randomization (5 studies) or because the target BP was arbitrarily described as an appropriate target $\mathrm{BP}$ according to the treating physician (1 study). Methodologically flawed RCTs were excluded because they were of a lower quality and level of evidence compared to some of the well-designed cohort studies that we evaluated. Other reasons for exclusion were that the study involved nonpharmacological interventions (e.g., coffee and cigarette smoking or concurrent hemodialysis) ${ }^{2}$ or that the study was a follow-up of patients who had already been treated for a hypertensive emergency or urgency $^{1}$ or who had had a run-in period with other drugs (1 study), making the interpretation of the results very difficult in terms of the drug of interest. Nineteen trials met the criteria for Level 1 or 2 evidence. The 4 hypertensive emergency and 15 hypertensive urgency studies represented 236 and 1,074 patients, respectively.

Eight of the 19 trials included in this review were open label or did not mention if the trial was 
blinded. ${ }^{8-10,16,17,19,23,24}$ We included these studies because they met our review's inclusion of Evidence Level 1 or 2 . In addition, the number of studies of this quality is limited and the further exclusion of studies would further limit the conclusions of this review.

The definitions of hypertensive emergencies and urgencies did vary in the studies with respect to specific BP measurements, but emergencies always involved hypertension with target end-organ damage, and urgencies without such damage. Moreover, measures of outcome were not uniform between studies. Some studies used the diastolic blood pressure (DBP) as the endpoint to indicate success, and either used a specific blood pressure (usually 95 to 110 $\mathrm{mm} \mathrm{Hg}),{ }^{8}$ a percentage reduction in blood pressure ${ }^{20}$ or a numeric $(20 \mathrm{~mm} \mathrm{Hg})^{13,20}$ fall in the DBP. Fewer studies used the systolic blood pressure (SBP) as the goal.

Many of the studies included in this review used adverse effects as outcome measures as well. None of the studies used immediate or long-term mortality endpoints. In addition, in the hypertensive emergency studies, resolution of end-organ dysfunction did not figure prominently as outcome measures in all the studies.

\section{Treatment for Hypertensive Emergencies}

We were unable to identify any prospective studies that addressed the questions of how quickly BP should be controlled in a hypertensive emergency or when maintenance therapy with antihypertensive medications should begin. We identified 3 small, level $2 \mathrm{~b}$ trials and one level $1 \mathrm{~b}$ trial that compared various therapies in patients with hypertensive emergencies (Table 2). ${ }^{8,10,21,23}$ Each of the 3 level $2 \mathrm{~b}$ trials used different entry criteria. One study included patients with increased SBP and/or increased DBP and any evidence of target end-organ damage. ${ }^{8}$ They found that nitroprusside led to a faster response (49\%) than did urapidil (20\%) in the first 15 minutes of therapy $(P<.001)$. However, due to the short half-life of nitroprusside, this difference was not seen at 4 hours. ${ }^{8}$ Nitroprusside was associated with more adverse side effects, including 2 episodes of hypotension, although none of them was associated with clinical sequelae $(23 \%$ vs $11 \%$; $P<.04)$. In addition, nitroprusside requires invasive monitoring. Another study included patients with an elevated DBP and hypertensive retinopathy and found that nitroprusside achieved the target BP more slowly than did nifedipine, ${ }^{10}$ while a third study included patients with DBP $>120 \mathrm{~mm}$ $\mathrm{Hg}$ but did not provide explicit information about target organ damage. ${ }^{23}$ This last study looked at 120 patients who were randomized to 1 of 4 treatment groups including: $10 \mathrm{mg}$ nifedipine SL; $50 \mathrm{mg}$ captopril SL; $0.15 \mathrm{mg}$ clonidine IM; or $10 \mathrm{mg}$ nifedipine SL and $40 \mathrm{mg}$ furosemide IV. No significant blood pressure differences were found between the 4 groups after treatment.

The last study, by Angeli et al., found that 7 of 10 patients treated with captopril or 5 of 10 treated with nifedipine were complete responders (not significant). ${ }^{21}$
This study also found that the duration of action for the 2 drugs was similar.

\section{Treatment for Hypertensive Urgencies}

We were unable to identify any high-quality studies that addressed what blood pressure defines a hypertensive urgency, how quickly blood pressure should be decreased in a hypertensive urgency, when maintenance therapy should be started, or whether patients with hypertensive urgencies should be treated in observed settings. We found 15 prospective trials representing levels $1 \mathrm{~b}$ and $2 \mathrm{~b}$ evidence that addressed therapy in patients with hypertensive urgencies (Table 3). Although many of these studies defined hypertensive urgencies differently, the most consistently used definition was a DBP of $>120 \mathrm{~mm} \mathrm{Hg}$. Methodological problems in the trials included small sample size, ${ }^{11,13,14,16,25}$ open label design, ${ }^{9,16,17,19,24}$ lack of follow-up in most of the studies, and contamination. ${ }^{14}$ In addition, few studies looked at outcomes more than 24 hours after randomization, and follow-up ranged from only 15 minutes to 1 week. Moreover, the trials used various definitions of "therapeutic response" and none looked at long-term blood pressure control or important cardiovascular endpoints.

In one study comparing nicardipine to placebo, nicardipine therapy led to effective blood pressure control in $65 \%$ of patients compared to $22 \%$ of patients in the placebo group $(P=.002) .{ }^{26}$ This is one of the few therapies that have been evaluated in a randomized control trial, and demonstrated statistical superiority over placebo. ${ }^{26}$ In another study, nifedipine was more effective at lowering the SBP at 30 minutes when compared with captopril, clonidine, and furosemide $(P<.02)$, but this difference was no longer seen after 30 minutes. ${ }^{11}$ Three dosage regimens of labetalol were compared in another study and differed only in their response at 2 hours, when the 200-mg dose was associated with significantly less tachycardia than either the 100-mg or 300-mg doses. ${ }^{20}$ In a study comparing long- and short-acting calcium channel blockers, long-acting lacidipine was more effective than nifedipine at keeping the blood pressure controlled at 24 hours $(P=.001) .^{25}$ In addition, 1 patient had a stroke syndrome 30 minutes after taking nifedipine (see Table 3). When compared with clonidine, nifedipine controlled blood pressure significantly more quickly. ${ }^{12}$ In a study by Rutledge et al., enalaprilat was significantly better at reducing blood pressure than placebo in the moderate hypertension group defined arbitrarily as a DBP of 100 to $114 \mathrm{~mm} \mathrm{Hg}^{14}$ Interestingly, 58\% of patients assigned to the placebo group responded to hospitalization and no active medications. When enalaprilat was compared with furosemide, the 2 agents did not differ in terms of efficacy.

Hirschl et al. showed that urapidil had a significantly higher rate of response when compared with nifedipine after a single dose $(92 \%$ vs $70 \% ; P<.04)$. This response was 


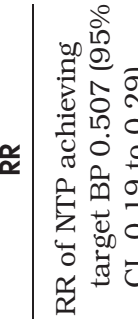

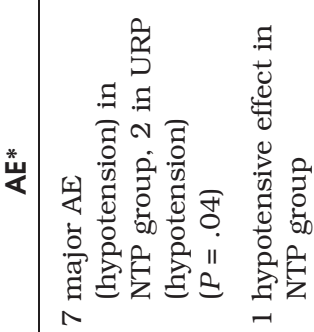

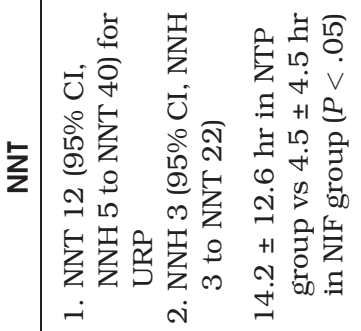

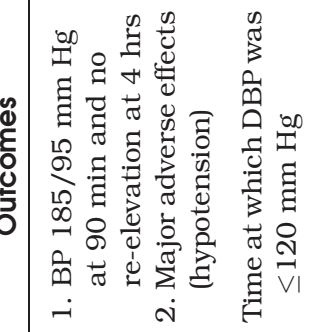
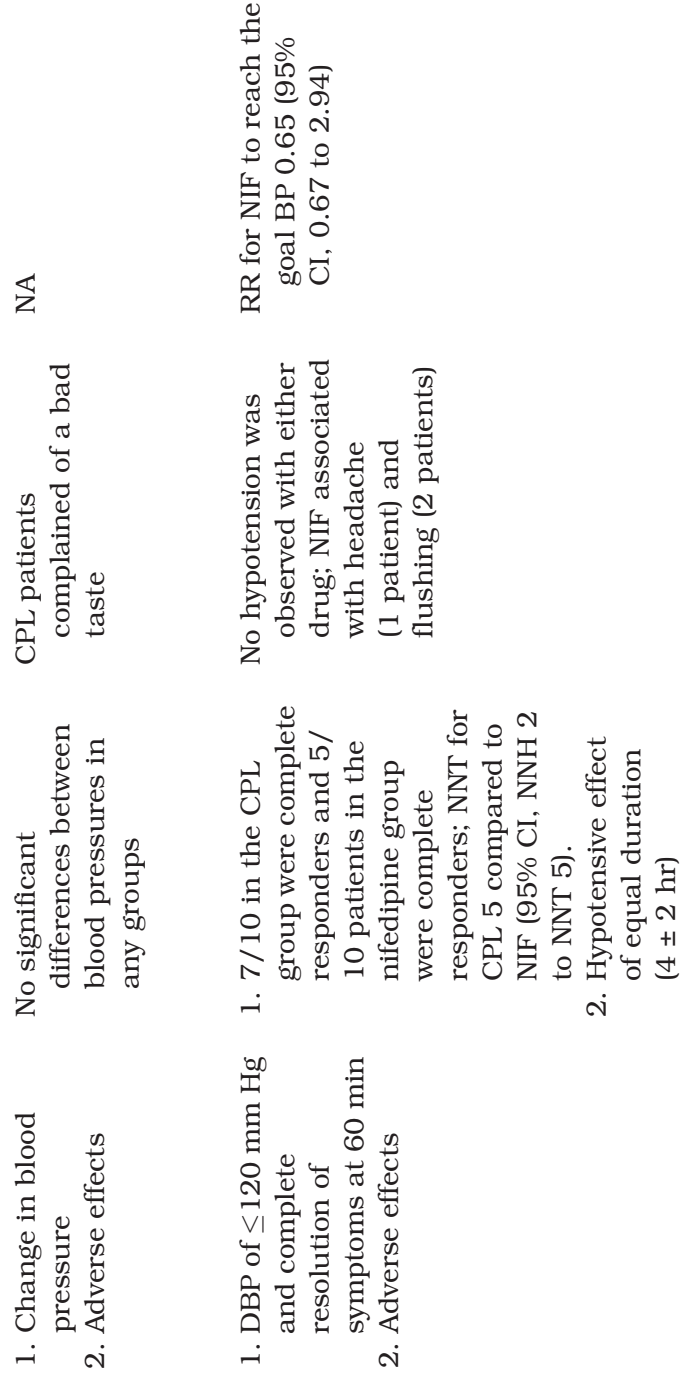



เด ะ

늘 놀

苯 on

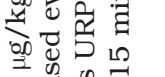

เి

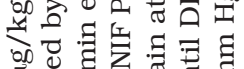

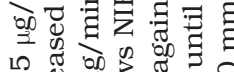

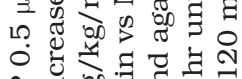
定吕寻

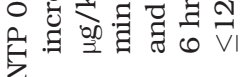

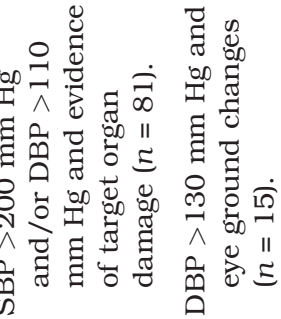

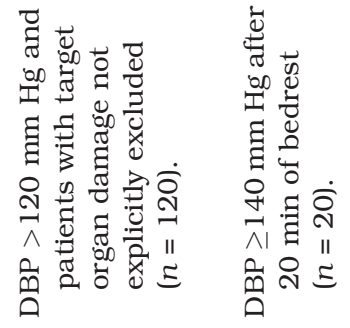

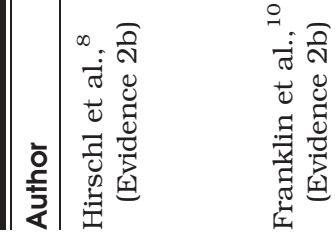

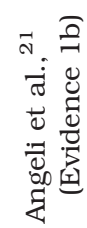

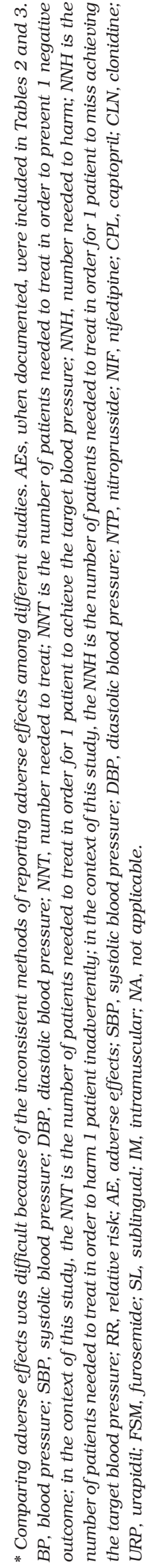


$100 \%$ and $50 \%$, in the urapidil and nifedipine groups, respectively, after a second dose in those patients who did not respond to the first dose. ${ }^{24}$ In addition, urapidil was associated with a shorter stay in the emergency room (83 vs 113 minutes; $P<.05$ ). The studies that compared oral nifedipine with oral labetalol, and oral nifedipine with oral nitrendipine found the agents to be of similar efficacy. ${ }^{15,16}$

In the 3 studies that examined fenoldopam in comparison to nitroprusside, the drugs were of comparable efficacy and all resulted in the achievement of the target BP in either the initial infusion phase or the maintenance phase (depending on the trial). ${ }^{17-19}$ The agents also had similar adverse event profiles. Nitroprusside was found to have an accumulation of thiocyanate metabolites, but did not result in clinical toxicity. ${ }^{19}$

In the study by Hirschl et al., ${ }^{9}$ urapidil was found to be almost uniformly successful when compared to the $70 \%$ to $72 \%$ response rates in the nifedipine or enalaprilat groups (NNT for urapidil of 4). In addition, the RR for nifedipine or enalaprilat to reach the target BP compared to urapidil was significantly less than 1. One nifedipine patient in this study had a transient ischemic attack (TIA). In the study by Wallin et al., the second placebo-controlled trial involving nicardipine concluded that the drug is a more successful antihypertensive than placebo, ${ }^{22}$ but this study involved both hypertensive urgencies and emergencies, as opposed to the study by Habib et al., which involved only hypertensive urgencies. ${ }^{26}$

\section{DISCUSSION}

After reviewing all of the available evidence, the best choice of hypertensive agent in urgencies and emergencies remains unclear. In emergencies, the most desirable NNT is for urapidil, ${ }^{8}$ although nitroprusside, captopril, and clonidine are likely acceptable choices as well. Comparing nitroprusside and urapidil with captopril and clonidine is difficult because no head-to-head studies have ever been done with these agents.

Hypertensive urgencies can also be treated with a variety of agents, including nicardipine, ${ }^{26}$ lacidipine, ${ }^{25}$ and urapidil, ${ }^{9}$ and nitroprusside or fenoldopam, ${ }^{17-19}$ which have the most favorable NNT profiles. Nifedipine can be used, but has rapid blood pressure-lowering properties that are not necessary in this nonemergent situation. This agent therefore shouldn't be used in the treatment of urgencies. In addition, nifedipine was associated with a TIA in 2 hypertensive urgency studies, ${ }^{9,25}$ and has been implicated by others as a cause of cardiovascular morbidity and mortality. ${ }^{27}$ No other antihypertensive agent in the studies we reviewed was associated with this complication. This association has been debated in medical literature and is still of uncertain significance. ${ }^{28,29}$

These recommendations must be viewed with caution and are meant to show what is known, and what is not known, about hypertensive urgencies and emergencies. The studies included in this review have many limitations.
First, tremendous variation and inconsistency exists in the definitions and cutoffs for urgencies and emergencies and for target blood pressures. Second, long-term outcomes were not well studied, and important clinical outcomes were often not measured. Third, studies were often underpowered, leading to wide confidence intervals with respect to treatment efficacy. Further, as demonstrated in Tables 2 and 3, the confidence intervals were so wide that they gave both NNT and number of patients needed to harm (NNH) data, indicating that the various agents may have either harmed or benefited patients. In addition, the small numbers of patients in the studies limited their power to detect differences in mortality and morbidity, and may also account for some of the inconsistencies found in the results. Finally, the reporting of adverse effects was not consistent, making comparison of adverse effects difficult (Tables 2 and 3), and the small study sizes may also have limited the ability to detect important differences in adverse effect profiles.

When faced with hypertensive urgencies and emergencies, the clinician has to not only select an appropriate antihypertensive agent but also assess how rapidly the blood pressure must be lowered. Unfortunately, the literature does not have data to support one timetable over another. Therefore, clinical judgement must be used in order to set a goal for the rate of decline of blood pressure, as well as for the target blood pressure. Clinical practice guidelines for the management of hypertensive emergencies suggest that the mean arterial blood pressure be reduced by $\leq 25 \%$ within 2 hours and to $160 / 100 \mathrm{~mm} \mathrm{Hg}$ by 6 hours. $^{30-32}$ In hypertensive urgencies, the goal blood pressure should be achieved over hours to days. Avoiding excessive reductions in blood pressure is advised because this can precipitate renal, cerebral, or coronary ischemia. $^{9,25}$ Frequent monitoring of blood pressure response to treatment (every 15 to 30 minutes) is also recommended. As some authors have pointed out, ${ }^{8}$ the rate of blood pressure lowering should be considered in the context of the patient's clinical condition, and does have clinical significance; patients with an aortic dissection, for example, require more rapid blood pressure control, ${ }^{33-35}$ compared to a patient with a hypertensive emergency and cerebrovascular symptoms, where a sudden drop in blood pressure might be dangerous.

Finally, many important questions remain unanswered. Future studies need to be consistent with respect to their operational definitions and cutoffs for urgencies and emergencies and for target blood pressures. This may serve as a better guide for clinicians. Second, studies need to follow patients over a period of time long enough to gather outcome data, such as cardiovascular morbidity and mortality, reduction in the number of hospitalizations, and length of stay information. The studies included in this review are of limited value because they use the surrogate endpoint of blood pressure control. Third, it remains unknown as to when patients with hypertensive crises should start maintenance therapy after 


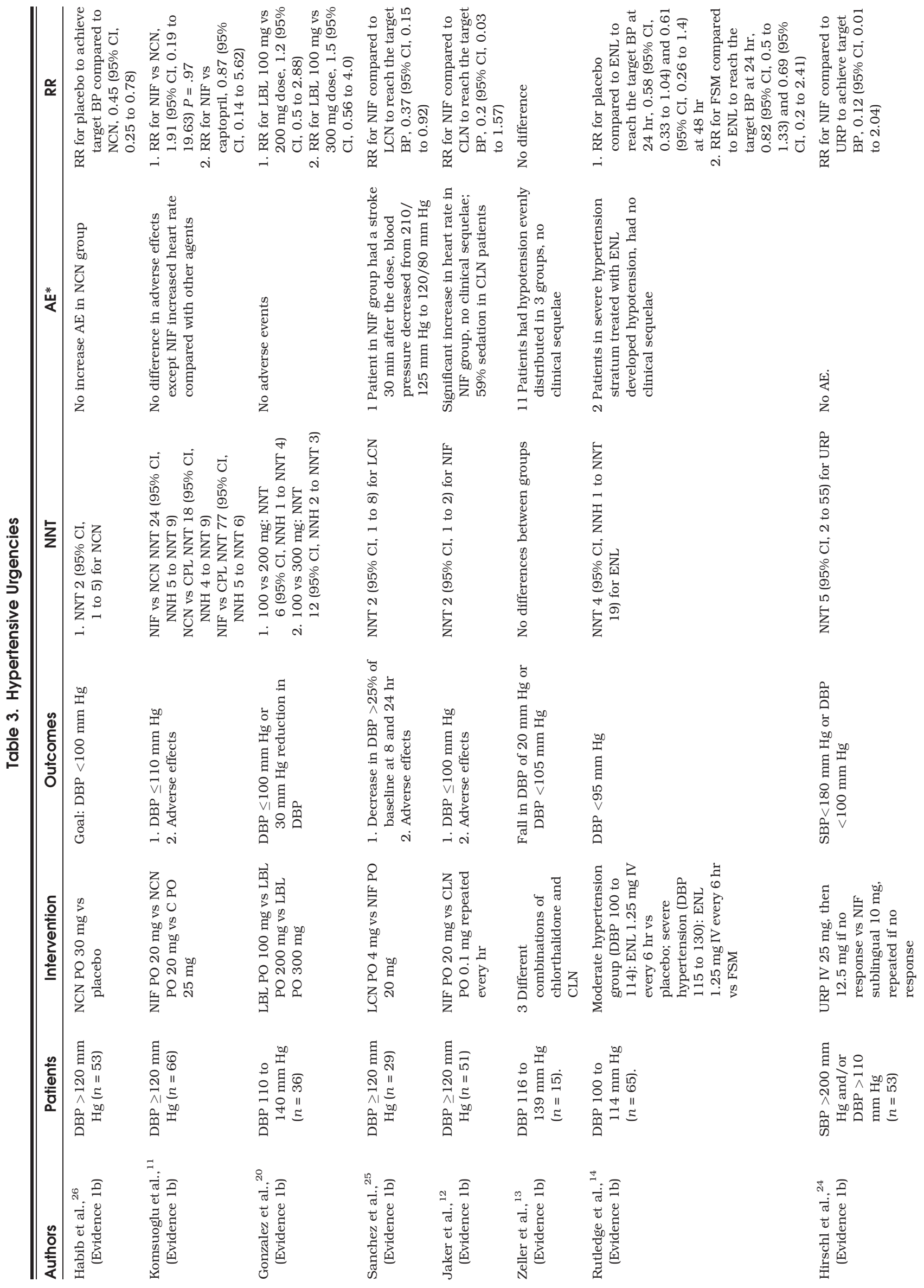




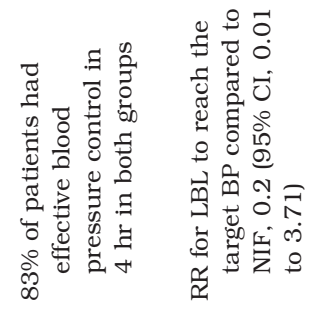

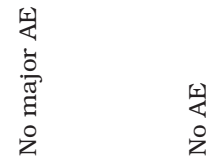

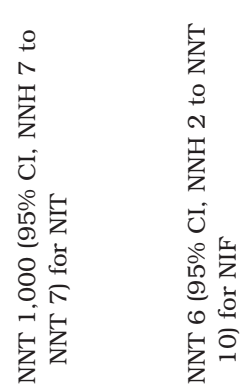

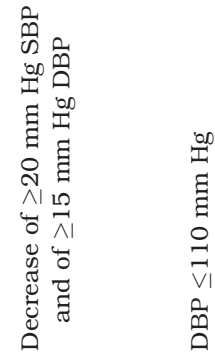

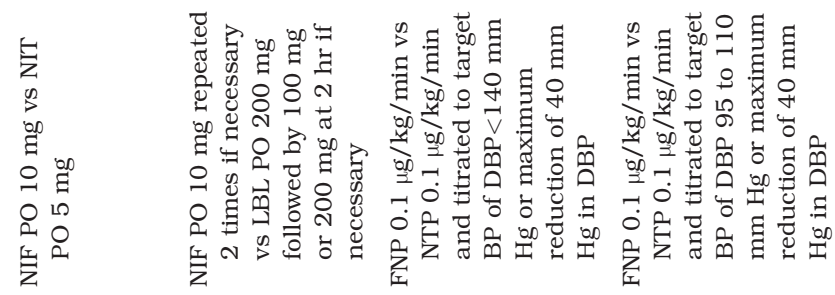

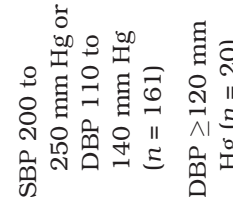

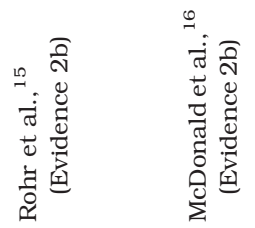

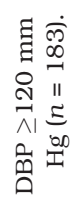

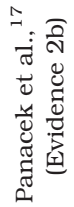

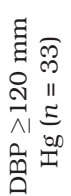

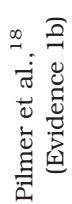

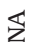

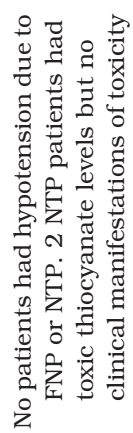

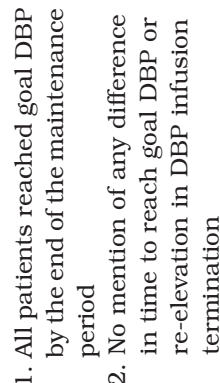

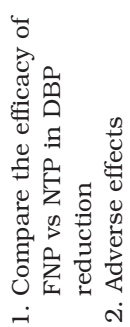

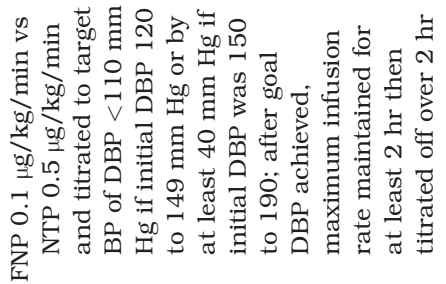

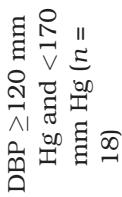

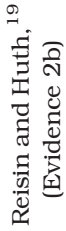




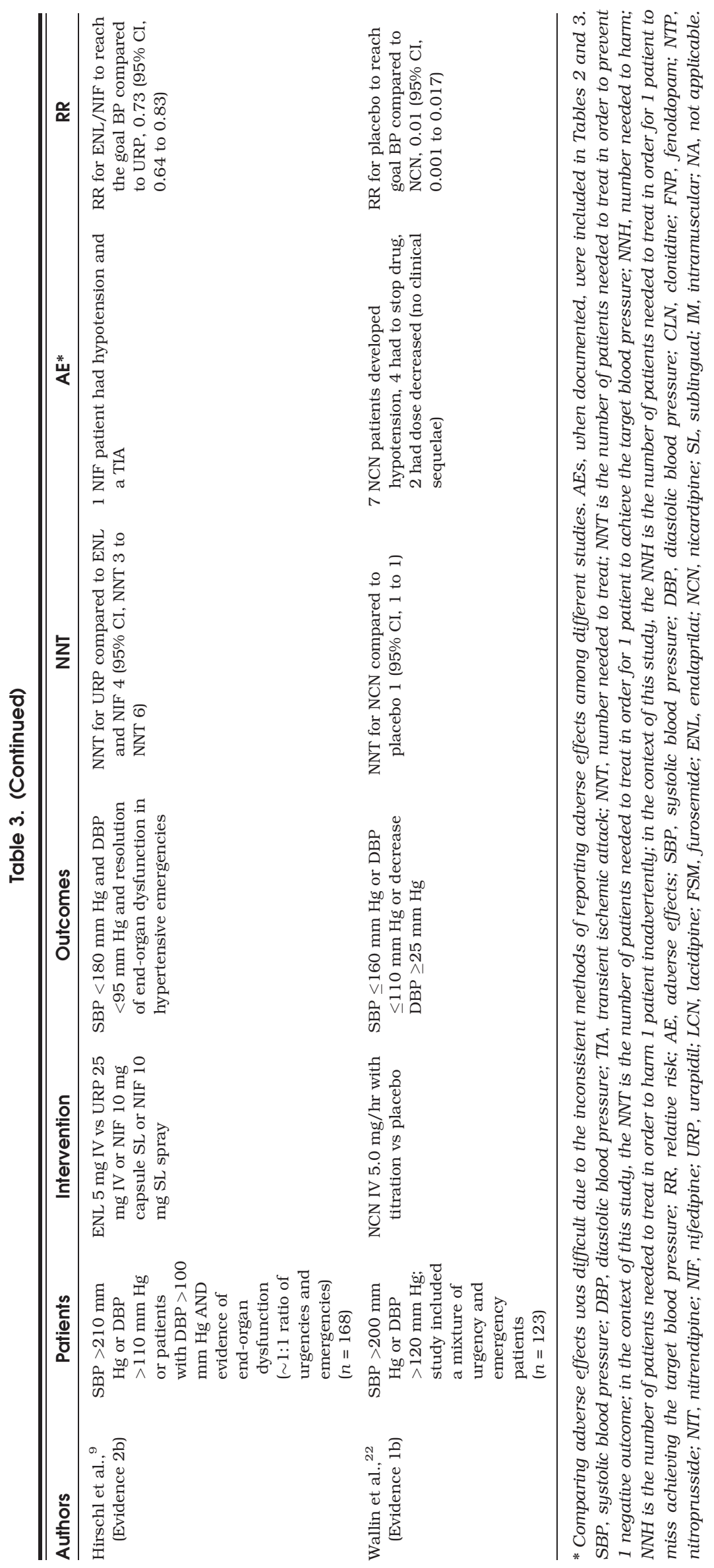


initial blood pressure control is accomplished in the emergency room. Another issue that has not been explored is how quickly the blood pressure should be lowered in this context. Finally, none of the studies we reviewed evaluated which patients should be admitted to the hospital and for how long, and which patients should be managed as outpatients. In addition, it is unclear whether these patients can be triaged outside of the emergency department in such settings as physicians' offices or over the phone. We believe that there is more research to be done in this area, and hope that future collaborative, multicenter, randomized double-blind controlled trials will be performed to address these issues.

\section{REFERENCES}

1. Kitiyakara C, Guzman N. Malignant hypertension and hypertensive emergencies. J Am Soc Nephrol. 1998;9:133-42.

2. Zampaglione B, Pascale C, Marchisio M, Cavallo-Perin P. Hypertensive urgencies and emergencies. Prevalence and clinical presentation. Hypertension. 1996;27:144-7.

3. Kincaid-Smith P, McMichael J, Murphy EA. The clinical course and pathology of hypertension with papilloedema (malignant hypertension). QJM. 1958;37:117-53.

4. Bechgaard P, Kopp H, Neilson J. One thousand hypertensive patients followed from 16-22 years. Acta Med Scand. 1956; 312(suppl): 175-83.

5. Haynes RB, Wilczynski C, McKibbon A, Walker C, Sinclair J. Developing optimal search strategies for detecting clinically sound studies in MEDLINE. J Am Med Inform Assoc. 1994;1:447-58.

6. Sackett DL, Straus S, Richardson WS, Rosenberg W, Hagro RB. Evidence-Based Medicine. How to Practice and Teach EvidenceBased Medicine. 2nd ed. London: Churchill Livingston; 2000.

7. Mount Sinai Hospital-University Health Network. 2000. Centre for Evidence Based Medicine: NNT Tables. Available at: http:// www.mtsinai.on.ca/masterframe.html. Accessed April 12, 2001.

8. Hirschl MM, Binder M, Bur A, et al. Safety and efficacy of urapidil and sodium nitroprusside in the treatment of hypertensive emergencies. Intensive Care Med. 1997;23:885-8.

9. Hirschl MM, Seidler D, Zeiner A, et al. Intravenous urapidil versus sublingual nifedipine in the treatment of hypertensive urgencies. Am J Emerg Med. 1993;11:653-6.

10. Franklin C, Nightengale S, Mambani B. A randomized comparison of nifedipine and sodium nitroprusside in severe hypertension. Chest. 1993;90:500-3.

11. Komsuoglu B, Sengun B, Bayram A, Komsuoglu SS. Treatment of hypertensive urgencies with oral nifedipine, nicardipine, and captopril. Angiology. 1991;42:447-54.

12. Jaker M, Atkin S, Soto M, Schmid G, Brosch F. Oral nifedipine vs. oral clonidine in the treatment of urgent hypertension. Arch Intern Med. 1989;149:260-5.

13. Zeller KR, Kuhnert LV, Matthews C. Rapid reduction of severe asymptomatic hypertension: a prospective, controlled trial. Arch Intern Med. 1989; 149:2186-9.

14. Rutledge J, Ayers C, Davidson R, et al. Effect of intravenous enalaprilat in moderate and severe hypertension. Am J Cardiol. 1988;62:1062-67.

15. Rohr G, Reimnitz P, Blanke P. Treatment of hypertensive emergency. Comparison of a new dosage form of the calcium antagonist nitrendipine with nifedipine capsules. Intensive Care Med. 1994; 20:268-71.
16. McDonald AJ, Yealy DM, Jacobson S. Oral labetalol vs. oral nifedipine in hypertensive emergencies in the emergency department. Am J Emerg Med. 1993;11:460-3.

17. Panacek EA, Bednarczyk EM, Dunbar LM, Foulke GE, Holcslaw TL. Randomized, prospective trial of fenoldopam vs. sodium nitroprusside in the treatment of acute severe hypertension. Acad Emerg Med. 1995;2:959-65.

18. Pilmer BL, Green JA, Panacek EA, et al. Fenoldopam mesylate versus sodium nitroprusside in the acute management of severe systemic hypertension. J Clin Pharmacol. 1993;33:549-53.

19. Reisin E, Huth M. Intravenous fenoldopam versus sodium nitroprusside in patients with severe hypertension. Hypertension. 1990;15(suppl I):I59-I62.

20. Gonzalez ER, Peterson MA, Racht EM, Ornato JP, Due DL. Doseresponse evaluation of oral labetalol in patients presenting to the emergency department with accelerated hypertension. Ann Emerg Med. 1991;20:333-8.

21. Angeli P, Chiesa M, Caregaro L, et al. Comparison of sublingual captopril and nifedipine in immediate treatment of hypertensive emergencies. A randomized, single-blind clinical trial. Arch Intern Med. 1991;151:678-82.

22. Wallin JD, Fletcher E, Ram CV, et al. Intravenous nicardipine for the treatment of severe hypertension: a double-blind, placebocontrolled multicenter trial. Arch Intern Med.1989; 149:2662-9.

23. Pascale C, Zampaglione B, Marchisio M. Management of hypertensive crisis: nifedipine in comparison with captopril, clonidine and furosemide. Curr Ther Res. 1992;51:9-18.

24. Hirschl MM, Seidler D, Mullner M, et al. Efficacy of different antihypertensive drugs in the emergency department. J Human Hypertension. 1996;24:1684-9.

25. Sanchez M, Sobrino J, Ribera L, Adrian AJ, Torres M, Coca A. Longacting lacidipine versus short-acting nifedipine in the treatment of acute asymptomatic acute blood pressure increase. J Cardiovasc Pharmacol. 1999;33:479-84.

26. Habib GB, Dunbar LM, Rodrigues R, Neale AC, Friday KJ. Evaluation of the efficacy and safety of oral nicardipine in the treatment of urgent hypertension: a multicenter, randomized, double-blind, parallel, placebo-controlled trial. Am Heart J. 1995; 129:917-23.

27. Psaty BM, Guralnik JM, Corti MC, et al. The risk of myocardial infarction associated with antihypertensive drug therapies. JAMA. 1995;274:620-5.

28. Epstein M. Calcium antagonists should continue to be first-line treatment of hypertension. Arch Intern Med. 1995;155:2150-6.

29. Messerli FH. Case-control study, meta-analysis and bouillabaisse: putting the calcium antagonist scare into context. Ann Intern Med. 1995; 123:888-9.

30. Ramsay LE, Williams B, Johnston GD, et al. for the British Hypertension Society. Guidelines for the management of hypertension: report of the Third Working Party of the British Hypertension Society. J Hum Hypertens. 1999; 13:569-92.

31. Feldman RD, Campbell N, Larochelle P, et al. for the Task Force for the Development of the 1999 Canadian Recommendations for the Management of Hypertension. 1999 Canadian recommendations for the management of hypertension. CMAJ. 1999;161(suppl 12): $1-17$.

32. The Sixth Report of the Joint National Committee on Prevention, Detection, Evaluation and Treatment of Hypertension. Arch Intern Med. 1997;157:2413-46.

33. DeSanctis RW, Doroghazi RM, Austen WG. Aortic dissection. N Engl J Med. 1987;317:1060-7.

34. Crawford ES. The diagnosis and management of aortic dissection. JAMA. 1990;264:2537-41.

35. Erbel R, Alfonso F, Boileau C, et al. Diagnosis and management of aortic dissection. Eur Heart J. 2001;22:1642-81. 